\title{
Signal processing for optimisation of low-powered GPR data with application in transportation engineering (roads and railways)
}

\author{
L. Bianchini Ciampoli, F. D’Amico \& A. Calvi \\ Department of Engineering, Roma Tre University, Rome, Italy
}

F. Benedetto

Signal processing for Telecommunications and Economics Lab., Roma Tre University

F. Tosti

School of Computing and Engineering, University of West London, London, United Kingdom

ABSTRACT: High-frequency air-coupled ground-penetrating radar (GPR) systems are used in road engineering for achieving high-resolution and fast imaging of the shallow layers of pavements. Regulatory policies on the permitted radiated power enacted by some international agencies for information and communication technologies, such as, the Federal Communications Commission operating in the United States, have led manufacturers to market low-powered GPR systems to comply with the standards.

The signal collected by these systems is more unstable than ordinary-powered GPRs, with the interpretation of the raw data being misleading or, mostly, totally subjective or even impossible. Thereby, the use of relevant signal processing techniques combined purposely within procedural schemes may help to reach reliability and effectiveness levels close to those granted by standard systems. In this study, a post-processing scheme aimed at maximising the correlation between signals collected by low-powered and standard $2 \mathrm{GHz}$ antenna systems in railway and road surveys is presented.

\section{INTRODUCTION}

Different forms of energy can be recognized within the electromagnetic spectrum, due to their wavelengths and frequencies. Frequencies ranging between $3 \mathrm{kHz}$ and $300 \mathrm{GHz}$ define the Radio Frequency (RF) part of the spectrum. Groundpenetrating radar (GPR) is an electromagnetic tool operating within the RF spectrum.

The RF spectrum composes part of the Non-ionizing radiations (NIR). The NIR radiative energy is able to only excite the ions contained within a medium when passing through it, instead of charging them. The most remarkable effect from this excitation is the heating of the medium. Other effects are generally referred to as non-thermal. The power of penetration of a NIR into a body, as well as its rate of absorption, is highly frequency-dependent (Kwan-Hoong 2003).
To date, the non-thermal effects of NIR on human health are not completely understood, and several international institutions payed attention on the free use of radiative instruments, especially in the case of uncontrolled environment, such as in GPR surveys of roads. This fact, as reported in many national and international research programmes (Dimbylow \& Bolch 2007, Gluszcz 2009, ICNRPI 1992, Health Protection Agency 2006, 2008, Mobile Telecommunications and Health Research Programme, 2007), is raising attention in the field. To this effect, several guidelines and standards have been published in the last decades, with the aim of regulating the use of NIR in both controlled and uncontrolled environments. Broadly, United States (US) and Western Europe refer to (IEEE Standards Coordinating Committee on Non-Ionizing Radiation Hazards 1992, ICNRPI
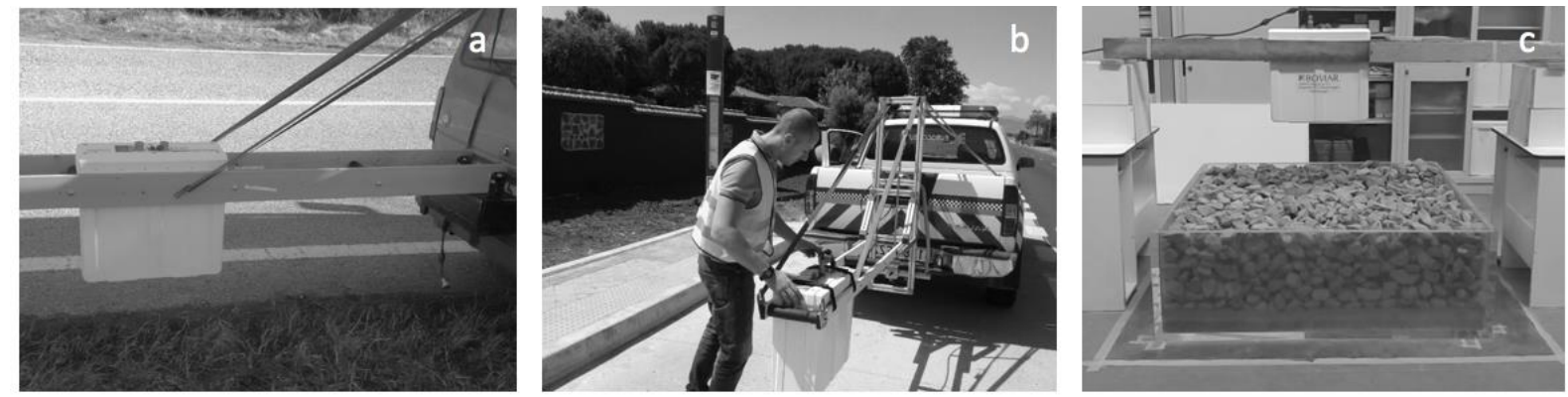

Figure 1. The three survey sites: road surveys conducted in the district of Rieti, Italy (a); road surveys conducted in the district of Guadalajara, Spain (b), Railway surveys conducted in laboratory at Roma Tre University (c). 
1992) whilst Russia, China, and Eastern Europe comply with (Sanitary Norms and Regulations. 1996). Typically, these science-based standards set a maximum permissible exposure (MPE) in terms of field strength, power density and time-averaged rate of energy transfer. Particularly, the US Federal Communications Commission (FCC) integrated the most common standards into a regulatory policy (FCC 1997).

More specifically in the area of GPR, manufacturers have to comply with different regulations on the power emission limit, and the challenge is mostly in countries like US where the threshold for maximum power emission is very low. As a consequence of a lower radiative power, these types of GPR systems exhibit worst performances in terms of signal-tonoise (SNR) ratio.

This work aims at evaluating the potential of lowpowered systems in civil engineering applications. In particular, a dataset of GPR signals have been gathered from surveys carried out on different transportation infrastructures with both standard and low-powered systems. In this study, the results coming from the different systems have been compared, and a postprocessing scheme for optimizing the information from the low-powered signals is proposed.Getting started

\section{THE DATASET}

In order to evaluate different conditions that are most likely to be encountered on site in transportation engineering applications, both road and railway GPR
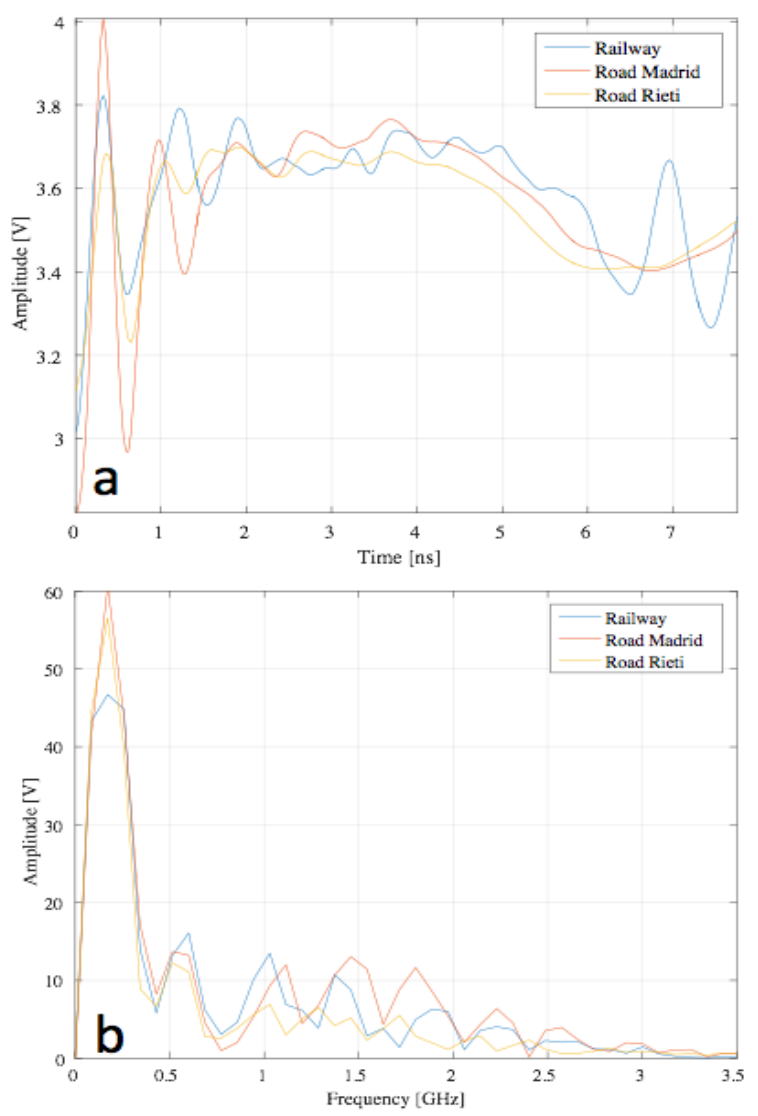
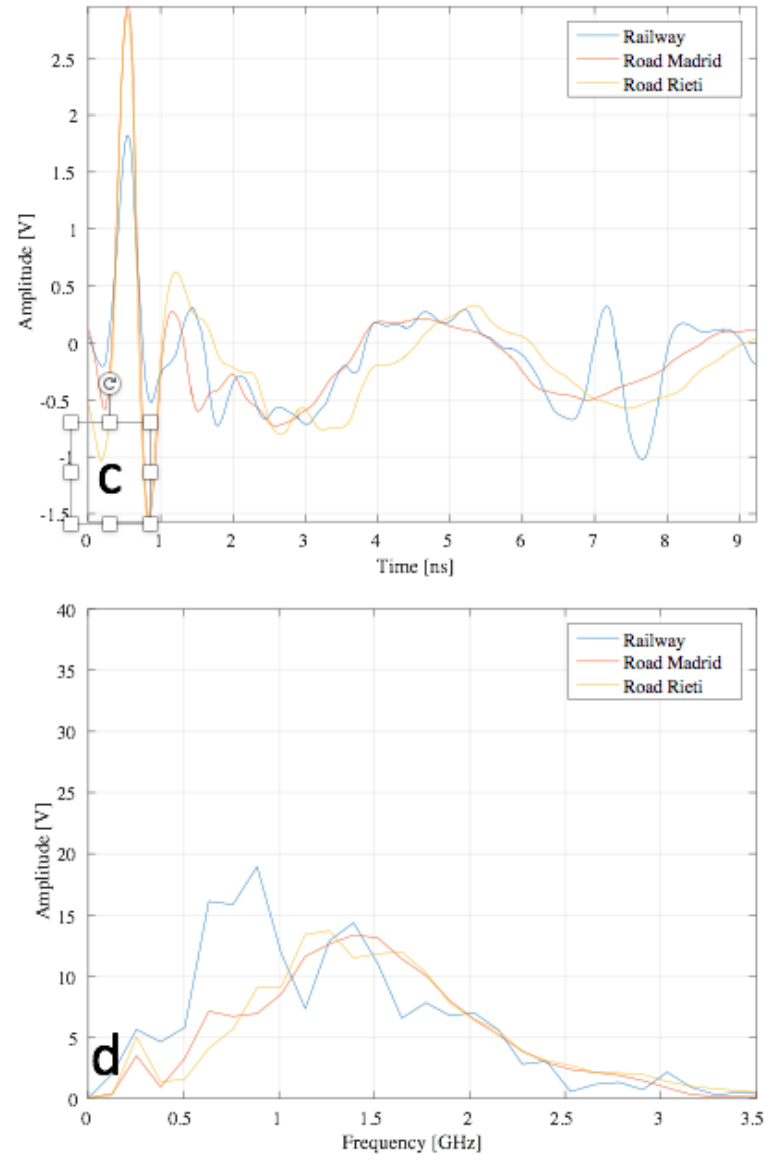

Figure 2. - Low-powered (a-b) and standard (b-c) raw GPR signals.

surveys have been performed. According to the scope of this study, the same road and railway sections have been surveyed by means of both standard and lowpowered systems. Pulsed GPR systems equipped with horn antennas and central frequency of $2000 \mathrm{MHz}$, were used. They were set to operate suspended in the air at $0.40 \mathrm{~m}$ height from the road/railway surface. The systems are manufactured by IDS Georadar and are actually identical in the whole set of components, with the exception of the radiative power. As far as the experimental frameworks are concerned, GPR surveys have been carried out at three different sites. In particular, road surveys have been performed over two different sections, located in the district of Rieti (Italy) and in the district of Guadalajara (Spain). In these surveys, the antenna was mounted onto an instrumented vehicle, and supported by a wooden framework. A third static data collection for railway engineering applications has been carried out in laboratory environment at Roma Tre University, over an experimental setup reproducing a ballasted railway track-bed. The data collections from the three survey sites are depicted in Figure 1. For sake of consistency, since the tests collected over the railway track-bed were static and, hence, produced a single A-scan, a single trace was also selected along the scanned sections for both the aforementioned road surveys. To ensure that the A-scans collected with the different 
GPR systems, are referred to the same geographical position, GPS coordinates have been matched.
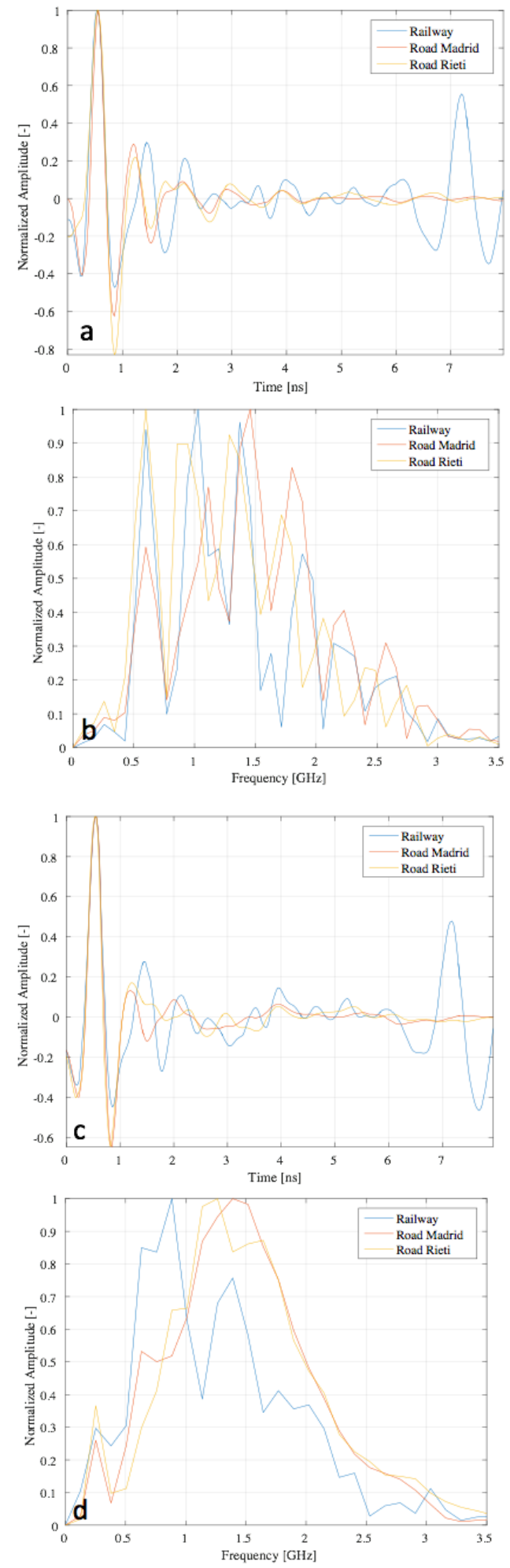

Figure 3. - Low-powered (a-b) and standard (b-c) GPR signals, after the standard band-pass filter application.

\section{DATA POST-PROCESSING}

Figure 2 shows the raw GPR traces, collected with the two antennas, both in the time and frequency domains. As clearly visible, low-powered signals are much more affected by noise, to the point that it is almost impossible to recognize the reflection peaks related to the dielectric discontinuities (Figure 2a). In more detail, it is worth noting the peaks of amplitude back-received at approximatively $200 \mathrm{MHz}$ in the low-powered spectra (Figure 2b), which are to be related with noise and tend to "blind" the signal contributes.

Thereby, the analysis of the raw data suggested that, by filtering out the lower frequency components

from the spectra, useful information could have been retrieved. Accordingly, after a preliminary zero- offset removal, a band-pass filter was applied to the whole dataset. As a common practice (Benedetto et al. 2016), the bandwidth included between the two cut-off frequencies has been set, in both cases, as 1.5 times the nominal frequency. As a result, the highpass and low-pass frequencies have been set as 500 $\mathrm{MHz}$ and $3500 \mathrm{MHz}$, respectively. For sake of comparison, the amplitude has been normalised. In Figure 3 , the effect of the band-pass filtering on the signals is shown. The benefit led by the procedure is clearly noticeable, especially for the low-powered case. It is now possible to recognize different amplitude peaks, deriving from the reflections at the interfaces of layers (Figure 3a). On the other hand, the low- powered spectra appear more chaotic than the regular ones, which is most likely due to the lower SNR ratio. Also, Figure $3 \mathrm{~b}$ still shows a low-frequency peak at 550 $\mathrm{MHz}$, hardly linkable with informative content. Accordingly, in order to maximise the likelihood between the two systems, a specific high-pass frequency needs to be set when processing low-powered data.

\section{POST-PROCESSING OPTIMISATION}

Using the data collected by GPR with standard radiated power as a reference, new band-pass filters were applied to the low-powered signals with differently

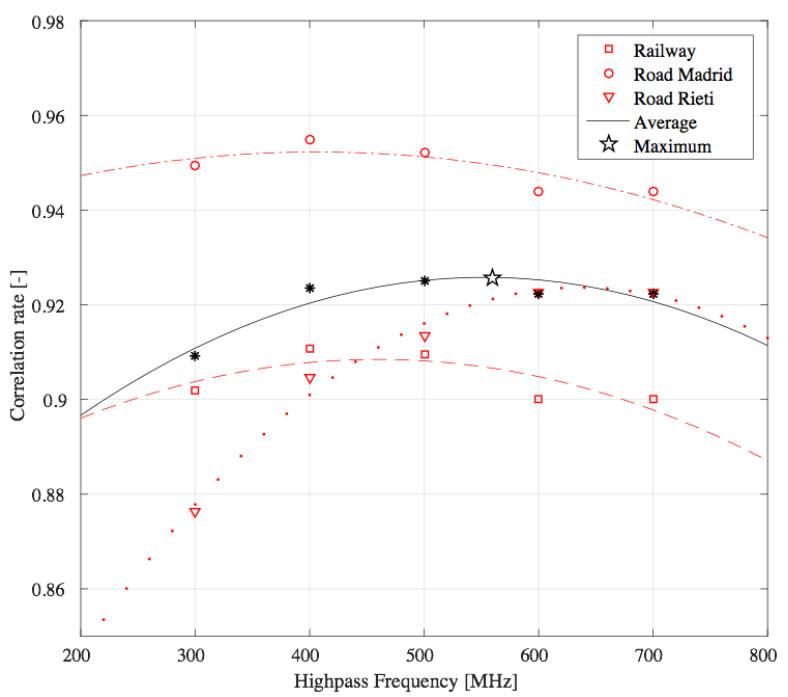

Figure 4. - Curves fitting the correlation coefficients calculated for different high-pass frequencies. 
set high-pass frequency values, in order to reach the
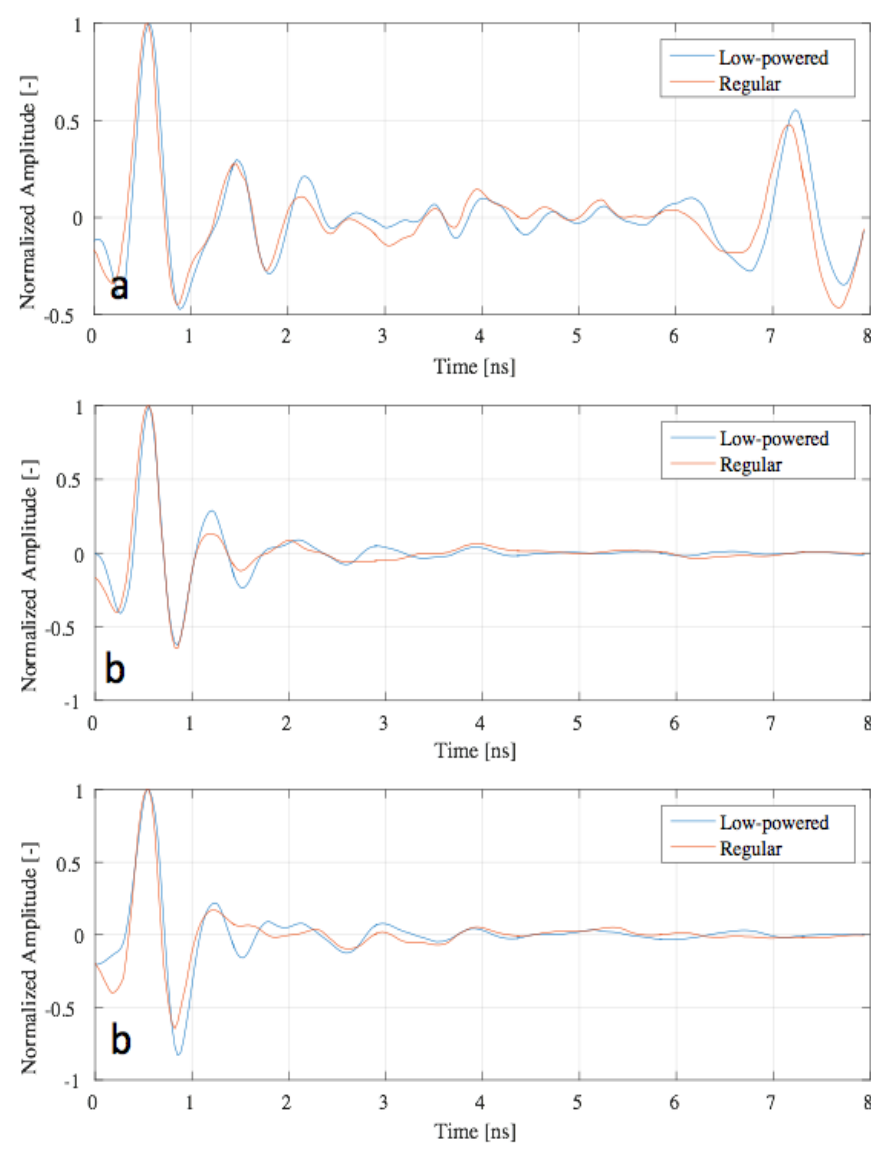

Figure 5. - Comparison between Low-powered and standard signals for Railway (a), Road Madrid (b) and Road Rieti (c) tests.

best matching between the data collected by the two systems. In particular, the range spanning between $200 \mathrm{MHz}$ and $700 \mathrm{MHz}$ has been tested, with steps of $100 \mathrm{MHz}$.

As reference parameter for the maximisation procedure, the correlation coefficient $(C C)$ between the signals collected with different systems was taken and calculated as:

$C C=\frac{\sigma_{x y}}{\sigma_{x} \sigma_{y}}$

with $X$ being the low-powered system signal, $Y$ being the standard system, $\sigma_{x}$ and $\sigma_{y}$ being their standard deviations, and $\sigma_{x y}$ being their covariance. For each test condition, five correlation rates have been computed, with respect to the five tested high-pass frequencies. The curves fitting these five values are representative of the effectiveness of the filter in reducing the discrepancy between the two signals.

In order to reach a processing scheme suitable for every conditions that can be tested in the transportation infrastructures surveys, a fourth curve, fitting the average correlation rates calculated for each processing condition, has been calculated. Accordingly, the frequency related to the maximum of this curve indicates the optimal high-pass frequency required to maximise the effectiveness of the low-powered sys- tems. The optimisation procedure is depicted in Figure 4, and a maximum band-pass frequency value of $560 \mathrm{MHz}$ is defined. This implies a shrinkage of the bandwidth from 1.5 to 1.45 times the central frequency. In Figure 5, instead, the comparison between the low-powered and standard processed signals is shown, for each test. As clearly visible, the application of such a scheme led to encouraging results.

\section{CONCLUSIONS AND FUTURE PERSPECTIVES}

This study deals with the performance analysis of low-powered GPR systems, equipped with horn antennas with central frequency of $2000 \mathrm{MHz}$. In order to evaluate the capability of such devices to detect targets in transportation engineering surveys, three experimental activities have been arranged for collecting GPR data with low-powered and standard systems, in both railway and road environments. The analysis of the raw data emphasized the need for using bandpass filters, to cut the noise contribution, which was very significant for the utilised low-powered system, especially at lower frequencies. Furthermore, the study highlighted that different filter bandwidths are required, whether the low-powered or the standard system are considered. Accordingly, by taking the standard signal as a reference, an optimum procedure has been developed for defining the best high-pass frequency to use in low-powered systems. The main purpose of this was to minimize the discrepancy between the signals, and maximising the effectiveness of low-powered antennas. As a result, an optimal high-pass frequency value of $560 \mathrm{MHz}$ was defined.

Future efforts in the field are to be focused on a similar identification of the high-pass frequency for GPR systems with different central frequencies, as well as on widening the range of test conditions and reaching a higher statistical significance.

\section{ACKNOWLEDGEMENTS}

The authors would like to express their gratitude to $\mathrm{Mr}$ Spartaco Cera, from Roma Tre University, for the valuable support given during the field activities. Special thanks to IDS Georadar for the precious collaboration in supplying part of the radar equipment. This work has also benefitted of the activities carried out within the EU funded COST Action TU1208 "Civil Engineering Applications of Ground Penetrating Radar." 


\section{REFERENCES}

Benedetto, A., Tosti, F., Bianchini Ciampoli, L., D’Amico, F. 2016. An overview of ground-penetrating radar signal processing techniques for road inspections, Signal Processing, In press.

Dimbylow, P. \& Bolch, W. 2007. Whole-body-averaged SAR from $50 \mathrm{MHz}$ to $4 \mathrm{GHz}$ in the University of Florida child voxel phantoms. Physics in Medicine and Biology, 52:66396649.

Federal Communications Commission Office of Engineering \& Technology. 1997. Evaluating Compliance with FCC Guidelines for Human Exposure to Radiofrequency Electromagnetic Fields, OET Bulletin 65, Edition 97-01.

Federal Office for Radiation Protection. DMF, German Mobile Telecommunication Research Programme. 2008., health risk assessment of mobile communications. BfS-SG-08/08. Bremerhaven: Verlag fu $\ddot{r}$ neue Wissenschaft GmbH.

Gluszcz, M. 1979. Difficulties in the certification of microwave disease. Med Przemyslowa, 30:147-150

Health Protection Agency. 2006. Power frequency electromagnetic fields, melatonin and the risk of breast cancer. Report of an independent Advisory Group on Non-Ionising Radiation. Chilton: HPA; Doc HPA, RCE-1.

Health Protection Agency. 2008. Static magnetic fields. Report of an independent Advisory Group on Non Ionising Radiation. Chilton: HPA; Doc HPA, RCE-6.

IEEE Standards Coordinating Committee on Non-Ionizing Radiation Hazards. 1992. Standard for Safe Levels With Re spect to Human Exposure to Radio Frequency Electromagnetic Fields, $3 \mathrm{KHz}$ to $300 \mathrm{GHz}$ (ANSI/IEEE, 1999), The Institute of Electrical and Electronics Engineers, New York.

Kwan-Hoong, N.g. 2003. Non-Ionizing Radiations-Sources, Biological Effects, Emissions and Exposures, Proceedings of the International Conference on Non-Ionizing Radiation at UNITEN (ICNIR2003), Electromagnetic Fields and Our Health, 20th-22nd October 2003.

International Commission on Non-Ionizing Radiation Protection (ICNRPI). 1996. Health issues related to the use of hand-held radiotelephones and base transmitters. Health Physics 70:587-593

Mobile Telecommunications and Health Research Programme. 2007. Report 2007. Available at: http://www. mthr.org.uk/documents/MTHR_report_2007.pdf.

Sanitary Norms and Regulations. 1996. Radiofrequency Electromagnetic Radiation (Rf Emr) Under Occupational and Living Conditions, Sanitary Norms and Regulations 2.2.4/2.1.8.055-96. 